\title{
Article \\ Parallel Locally Strictly Convex Surfaces in Four-Dimensional Affine Space Contained in Hyperquadrics
}

\author{
Paweł Witowicz \\ The Faculty of Mathematics and Applied Physics, Rzeszów University of Technology, al. Powstańcow \\ Warszawy 12, 35-959 Rzeszów, Poland; witowicz@prz.edu.pl
}

check for updates

Citation: Witowicz, P. Parallel Locally Strictly Convex Surfaces in Four-Dimensional Affine Space Contained in Hyperquadrics. Symmetry 2021, 13, 1575. https:// doi.org/10.3390/sym13091575

Academic Editor: José Carlos R. Alcantud

Received: 3 August 2021

Accepted: 24 August 2021

Published: 26 August 2021

Publisher's Note: MDPI stays neutral with regard to jurisdictional claims in published maps and institutional affiliations.

Copyright: (C) 2021 by the author. Licensee MDPI, Basel, Switzerland. This article is an open access article distributed under the terms and conditions of the Creative Commons Attribution (CC BY) license (https:// creativecommons.org/licenses/by/ $4.0 /)$.

\begin{abstract}
Locally strictly convex surfaces in four-dimensional affine space are studied from a perspective of the affine structure invented by Nuño-Ballesteros and Sánchez, which is especially suitable in convex geometry. The surfaces that are embedded in locally strictly convex hyperquadrics are classified under assumptions that the second fundamental form is parallel with respect to the induced connection and the normal connection is compatible with a metric on the transversal bundle. Both connections are induced by a canonical transversal plane bundle, which is defined by certain symmetry conditions. The obtained surfaces are always products of an ellipse and a conical planar curve.
\end{abstract}

Keywords: affine metric; strictly convex surface; symmetric equiaffine transversal bundle; antisymmetric equiaffine transversal bundle; hyperquadric

\section{Introduction}

The main purpose of this paper is to characterize a class of surfaces embedded in locally strictly convex hyperquadrics contained in the four-dimensional affine space. Every such surface is locally strictly convex itself because it locally lies on one side of a tangent space of the hyperquadric and has a non-degenerate contact with it.

In Section 2, we recall a notion of a locally strictly convex surface. Every point of such a surface has a neighborhood that lies on one side of a supporting hyperplane, which is tangent to the surface. We also recall basic equations of surfaces of codimension two.

In Section 3, we prove that there is a collection of supporting hyperplanes that vary smoothly on an oriented, locally strictly convex surface (compare [1] in the Euclidean case). Simultaneously, we explain the existence of a smooth transversal vector field, which, together with the tangent plane, spans a supporting hyperplane at every point of the surface. Such a transversal field is called a metric field.

In order to study natural geometric properties, we choose a geometric structure that was invented by Nuño-Ballestero and Sánchez in [2]. The construction starts with a family of metric fields that define affine metrics. Those metrics are definite on an oriented, locally strongly convex surface (unlike the Burstin-Meyer metric, see [3]), [4]. For every metric of the family, two canonical equiaffine transversal bundles, a symmetric and an antisymmetric one are defined in [2]. A transversal bundle is called equiaffine if the metric volume element is parallel with respect to the affine connection induced on the surface by the bundle. We recall briefly these concepts in Section 4.

If the surface is embedded in a locally strictly convex hyperquadric then there is a metric from the aforementioned family that coincides with the Blaschke metric induced from the hyperquadric. This special choice of a metric implies that both equiaffine transversal bundles coincide. Moreover, the Blaschke normal of the hyperquadric belongs to them, which meets our expectations, but it is not generally true for other structures, for example the well-known Nomizu-Vrancken affine normal bundle defined in [4]. In Section 5, we give a complete proof of the last property because there is a certain omission in its proof in [2]. The property itself is so important that it justifies the whole construction. 
In Section 6, we prove the main theorem of the paper (Theorem 4) based on the affine structure invented in [2]. We classify surfaces embedded in locally strictly convex hyperquadrics of $\mathbb{R}^{4}$ under the assumptions that the cubic form vanishes (such submanifolds are called parallel because the second fundamental form is parallel with respect to the induced connections) and a normal connection is compatible with a metric canonically defined on the transversal bundle. We prove that if a surface satisfies the above properties and has no inflection points, then it is locally affine equivalent to an open part of one of three types of surfaces: a product of two ellipses (affine equivalent to the Clifford torus), the product of an ellipse and a hyperbola or the product of an ellipse and a parabola. Thus, we obtained another affine characterization of the Clifford torus as a parallel locally strictly convex surface contained in the ellipsoid (see Opozda's work [5]). We note that in [6], a topological torus is characterized as a compact surface with the indefinite Burstin-Mayer metric.

\section{Preliminaries}

In this section, we assume that $M$ is a connected surface immersed in $\mathbb{R}^{4}$ by a smooth mapping $f: M \rightarrow \mathbb{R}^{4}$ and that there is a transversal plane bundle $\sigma$ such that $f_{*}\left(T_{x} M\right) \oplus$ $\sigma_{x}=\mathbb{R}^{4}$ for every $x \in M$. Because of the local character of this work, we can restrict ourselves to embeddings, and we will write:

$$
T_{x} M \oplus \sigma_{x}=\mathbb{R}^{4} .
$$

Definition 1. Let $M$ be a surface embedded in $\mathbb{R}^{4}$ and let $p \in M$. M is locally strictly convex at $p$ if there is a hyperplane $\pi_{p}$ tangent to $M$ at $p$, having a non-degenerate contact of order one with $M$ and an open neighborhood $U$ of $p$ such that $U \backslash\{p\}$ lies on one side of $\pi_{p}$. We say that $\pi_{p}$ is a non-singular support hyperplane of $U$. The contact of order one means that the second derivative of any regular curve on $M$ passing through $p$ is transversal to $\pi_{p}$ at $p$.

Remark 1. We note that for a given non-singular support hyperplane and an arbitrary transversal plane, there is a vector in this plane which, together with the tangent space, spans the support hyperplane.

We assume that $M$ is locally strictly convex. We study and develop a theory developed in [2]. Let $X, Y$ be arbitrary tangent vector fields and $\xi$ be a transversal vector field, which is a section of $\sigma$. Denoting by $D$ the standard linear connection in $\mathbb{R}^{4}$, we obtain the following decompositions:

$$
\begin{aligned}
& D_{X} Y=\nabla_{X} Y+h(X, Y), \\
& D_{X} \xi=-S_{\xi} X+\nabla_{X}^{\perp} \xi,
\end{aligned}
$$

where $\nabla_{X} Y$ and $-S_{X} \xi$ denote the tangent components, and $h(X, Y)$ and $\nabla_{X}^{\frac{1}{X}} \xi$ denote the transversal components of the left hand sides of the equations. It is well-known ([3]) that $\nabla$ is a torsion-free affine connection, $h$ is a $\mathcal{C}^{\infty}(M)$-bilinear, symmetric $\sigma$-valued mapping called the second fundamental form, and $S_{\xi}$ is a $(1,1)$ tensor field. We call $\nabla^{\perp}$ the normal connection. It is actually a connection in $\sigma$. Both connections are said to be induced by $\sigma$. For a given local basis $\left\{\xi_{1}, \xi_{2}\right\}$ of $\sigma$, we introduce the following notations for the components of $h$ and the normal connection:

$$
\begin{aligned}
h(X, Y) & =h^{1}(X, Y) \xi_{1}+h^{2}(X, Y) \xi_{2} \\
\nabla_{X}^{\perp} \xi_{j} & =\tau_{j}^{1}(X) \xi_{1}+\tau_{j}^{2}(X) \xi_{2}
\end{aligned}
$$


for $\mathrm{j}=1,2$. Thus, $h^{1}$ and $h^{2}$ are bilinear symmetric forms, and $\tau_{j}^{i}$ are one-forms. We will also abbreviate $S_{\xi_{j}}$ by $S_{j}$. It is proven in [4] that the following equations of Gauss, Codazzi and Ricci hold ( $X, Y$ and $Z$ denote tangent vector fields).

$$
\begin{aligned}
& R(X, Y) Z=h^{1}(Y, Z) S_{1} X+h^{2}(Y, Z) S_{2} X-h^{1}(X, Z) S_{1} Y-h^{2}(X, Z) S_{2} Y, \\
& \text { mappings }(X, Y, Z) \mapsto\left(\nabla_{X} h^{i}\right)(Y, Z)+\tau_{1}^{i}(X) h^{1}(Y, Z)+\tau_{2}^{i}(X) h^{2}(Y, Z) \\
& \text { are totally symmetric, } i=1,2 ; \\
& \left(\nabla_{X} S_{i}\right) Y-\left(\nabla_{Y} S_{i}\right) X=-\tau_{i}^{1}(Y) S_{1} X+\tau_{i}^{1}(X) S_{1} Y-\tau_{i}^{2}(Y) S_{2} X+\tau_{i}^{2}(X) S_{2} Y \\
& \text { for } i=1,2 ; \\
& h^{1}\left(X, S_{1} Y\right)-h^{1}\left(Y, S_{1} X\right)=d \tau_{2}^{1}(X, Y)+\tau_{1}^{2}(Y) \tau_{2}^{1}(X)-\tau_{2}^{1}(Y) \tau_{1}^{2}(X) \\
& h^{2}\left(X, S_{1} Y\right)-h^{2}\left(Y, S_{1} X\right)=d \tau_{1}^{2}(X, Y)+\tau_{1}^{1}(Y) \tau_{1}^{2}(X)-\tau_{1}^{2}(Y) \tau_{1}^{1}(X)+ \\
& \tau_{1}^{2}(Y) \tau_{2}^{2}(X)-\tau_{2}^{2}(Y) \tau_{1}^{2}(X) \\
& h^{2}\left(X, S_{2} Y\right)-h^{2}\left(Y, S_{2} X\right)=d \tau_{2}^{2}(X, Y)+\tau_{2}^{1}(Y) \tau_{1}^{2}(X)-\tau_{1}^{2}(Y) \tau_{2}^{1}(X) \\
& h^{1}\left(X, S_{2} Y\right)-h^{1}\left(Y, S_{2} X\right)=d \tau_{2}^{1}(X, Y)+\tau_{1}^{1}(X) \tau_{2}^{1}(Y)-\tau_{2}^{1}(X) \tau_{1}^{1}(Y)+ \\
& \tau_{2}^{1}(X) \tau_{2}^{2}(Y)-\tau_{2}^{2}(X) \tau_{2}^{1}(Y) .
\end{aligned}
$$

We also consider the cubic form $K$, which involves both connections:

$$
K(X, Y, Z)=\nabla_{X}^{\frac{1}{X}} h(Y, Z)-h\left(\nabla_{X} Y, Z\right)-h\left(Y, \nabla_{X} Z\right) .
$$

It is $\sigma$-valued and trilinear over $\mathcal{C}^{\infty}(M)$. Its components with respect to the frame $\left\{\xi_{1}, \xi_{2}\right\}$ are $(3,0)$ tensor fields, and they will be denoted by $K^{1}, K^{2}$, so the following formulas hold for $i=1,2$ :

$$
K^{i}(X, Y, Z)=\left(\nabla_{X} h^{i}\right)(Y, Z)+\tau_{1}^{i}(X) h^{1}(Y, Z)+\tau_{2}^{i}(X) h^{2}(Y, Z) .
$$

\section{Non-Singular Support Hyperplanes}

Let $M$ be a locally strictly convex surface in $\mathbb{R}^{4}$ with a transversal bundle $\sigma$. We fix a point $p \in M$. Let $\pi_{p}$ be a hyperplane passing through $p$. Let $\left(X_{1}\right)_{p},\left(X_{2}\right)_{p}$ be a basis of $T_{p} M$ and $\xi_{p} \in \sigma_{p}$ be a transversal vector at $p$ such that $\pi_{p}$ is spanned by $\left\{\left(X_{1}\right)_{p},\left(X_{2}\right)_{p}, \xi_{p}\right\}$ (see Remark 1). Define a function $H_{p}: M \rightarrow \mathbb{R}$ :

$$
H_{p}(x):=\left[\left(X_{1}\right)_{p},\left(X_{2}\right)_{p}, x-p, \xi_{p}\right],
$$

where $[., \ldots, \ldots]$ denotes the usual determinant in $\mathbb{R}^{4}$. It follows from Definition 1 that the hyperplane $\pi_{p}$ locally supports $M$ in a neighborhood of the point $p$ if and only if $H_{p}$ has a strict local extremum at $p$.

We notice that in the considered case, the differential $d H_{p}$ vanishes at $p$ so the problem of an extremum of $H_{p}$ at $p$ comes down to the Hessian of composition of $H_{p}$ with an arbitrary chart. If we choose a chart $(V, \phi)$ around a point $p$ with local coordinates $u_{1}, u_{2}$, the Hessian of $H_{p} \circ \phi^{-1}$ at $p$ acting on the standard basis $e_{1}, e_{2}$ in $\mathbb{R}^{2}$ is equal to:

$$
\operatorname{Hess}_{p}\left(H_{p} \circ \phi^{-1}\right)\left(e_{i}, e_{j}\right)=\left[\left(X_{1}\right)_{p},\left(X_{2}\right)_{p}, \frac{\partial^{2}\left(\phi^{-1}\right)}{\partial u_{i} \partial u_{j}}(\phi(p)), \xi_{p}\right]=\left[\left(X_{1}\right)_{p},\left(X_{2}\right)_{p},\left(D_{\partial_{i}} \partial_{j}\right)_{p}, \xi_{p}\right]
$$

where $\partial_{i}(p)=d_{\phi(p)}\left(\phi^{-1}\right)\left(e_{i}\right)$ and $i, j=1,2$. Let us consider a mapping

$$
\Psi_{p}: \mathfrak{X}(M) \times \mathfrak{X}(M) \ni(X, Y) \mapsto\left[\left(X_{1}\right)_{p},\left(X_{2}\right)_{p},\left(D_{X} Y\right)_{p}, \xi_{p}\right] \in \mathbb{R}
$$

which is bilinear over the ring of smooth functions on $M$ because $\Psi(X, f Y)=f \Psi(X, Y)$ as the determinant is independent of the tangent component of $D_{X}(f Y)$, where $f$ is a function. It is also symmetric because $D_{Y} X=D_{X} Y-[X, Y]$.

Therefore, we conclude that if mapping $\Psi_{p}$ is definite then, after fixing a local basis of tangent vector fields associated with a chart $\phi$, the matrix $\left[\Psi_{p}\left(\partial_{i}, \partial_{j}\right)\right]_{i j}$ is definite so 
the Hessian of $H_{p} \circ \phi^{-1}$ is definite. Consequently, $H_{p} \circ \phi^{-1}$ has an extremum and a nondegenerate critical point at $p$. This property does not depend on $\phi$. The converse is also true because of the bilinearity of $\Psi_{p}$. Therefore, we will say that $H_{p}$ has a non-degenerate critical point at $p$ if $\Psi_{p}$ is definite.

In the following proposition, we compare notions introduced above.

Proposition 1. Let $M,\left(X_{1}\right)_{p},\left(X_{2}\right)_{p}$ and $\xi_{p}$ be as above. The following conditions are equivalent:

(i) the hyperplane $\pi_{p}$ spanned by $\left\{\left(X_{1}\right)_{p},\left(X_{2}\right)_{p}, \xi_{p}\right\}$ has contact of order one with the surface $M$ at $p \in M$;

(ii) for every curve $\gamma$ on $M$ such that $\gamma(0)=p, \gamma^{\prime}(0) \neq 0$ and for every tangent vector field $X$ that extends locally vector field $\gamma^{\prime}(t), \Psi_{p}(X, X) \neq 0$;

(iii) the mapping $\Psi_{p}$ is definite;

(iv) the function $H_{p}$ has a local extremum and a non-degenerate critical point at $p$;

(v) the hyperplane $\pi_{p}$ spanned by $\left(X_{1}\right)_{p},\left(X_{2}\right)_{p}$ and $\xi_{p}$ is a non-singular hyperplane supporting a neighborhood of $p$ in $M$.

Proof. (i) $\Leftrightarrow(i i)$ Looking at (ii), we notice that $\Psi_{p}(X, X)$ depends only on the values of $\gamma^{\prime}(t)$ for $t$ in a neighborhood of 0 . Since

$$
\Psi_{p}(X, X)=\left[\left(X_{1}\right)_{p},\left(X_{2}\right)_{p}, D_{\gamma^{\prime}(0)} \gamma^{\prime}(t), \xi_{p}\right]=\left[\left(X_{1}\right)_{p},\left(X_{2}\right)_{p}, \gamma^{\prime \prime}(0), \xi_{p}\right],
$$

$\Psi_{p}(X, X) \neq 0$ if and only if $\gamma^{\prime \prime}(0)$ does not belong to $\pi_{p}$.

(ii) $\Rightarrow$ (iii) It is sufficient to prove that for every tangent vector field $X$ that is non-zero at $p, \Psi_{p}(X, X) \neq 0$. Let $\psi_{t}(x)$ be a one-parameter group of diffeomorphisms generated by $X$ in a neighborhood of $p$. Define a curve $\gamma(t):=\psi_{t}(p)$. Then, $X_{\gamma(t)}=\gamma^{\prime}(t)$ and $\left(D_{X} X\right)_{p}=\left(D_{X} X\right)_{\gamma(0)}=D_{\gamma^{\prime}(0)} \gamma^{\prime}(t)=\gamma^{\prime \prime}(0)$, and (ii) implies that $\Psi_{p}(X, X) \neq 0$.

(iii) $\Rightarrow$ (ii) It follows from the well-known possibility of extending a vector field from a curve to an open set of a surface.

(iii) $\Leftrightarrow$ (iv) It is a consequence of the fact that $d_{p} H_{p}=0$, as well as a definition of non-degeneracy of the critical point of $H_{p}$.

(iv) $\Rightarrow$ (v) Since $H_{p}(p)=0$, (iv) implies that $H_{p}$ has a constant sign in a neighborhood of $p$ in $M$, and, consequently, this neighborhood lies on one side of $\pi_{p}$. Applying (i), we deduce that $\pi_{p}$ is a non-singular supporting hyperplane for $U$.

Implication $(\mathrm{v}) \Rightarrow(\mathrm{i})$ is obvious.

In the next step, we discuss a question of the local existence of a smooth family of non-singular support hyperplanes for a locally strictly convex surface $M$. First, we construct a local extension of a transversal non-zero vector, which satisfies property $(\mathrm{v})$ of Proposition 1.

Lemma 1. Let $\eta \in \sigma_{p} \backslash\{0\}$ be a vector transversal to $M$. Then, there exists a local non-vanishing vector field $\xi$ in a certain neighborhood $U$ of $p$ such that for every $x \in U, \xi_{x} \in \sigma_{x}$ and $\xi_{p}=\eta$.

Proof. Let $V$ be such an open neighborhood of $p$ that there exists a tangent frame $\left\{X_{1}, X_{2}\right\}$ and a transversal frame $\left\{\xi_{1}, \xi_{2}\right\}$ in $V$. Hence, there are smooth functions $a, b, c$ and $d$ in $V$ such that $\eta=a X_{1}+b X_{2}+c \xi_{1}+d \xi_{2}$ and $a(p)=b(p)=0$. Then, $\xi:=c \xi_{1}+d \xi_{2}$ is a transversal vector field and $\xi_{p}=\eta \neq 0$, so $\xi \neq 0$ in a neighborhood $U$ of $p$. Thus, $\xi$ satisfies the assertions of the lemma in $U$.

Next, we show that a non-singular support hyperplane can be locally extended in a smooth way.

Proposition 2. Let $X_{1}, X_{2}$ be a tangent frame in a neighborhood of $p \in M$ and $\eta \in \sigma_{p}$ be such a vector that a hyperplane $\pi_{p}$ spanned by $\left\{\left(X_{1}\right)_{p},\left(X_{2}\right)_{p}, \eta\right\}$ is a non-singular local support hyperplane for $M$ at $p$. Then, there exists an open neighborhood $U$ of $p$ and a transversal vector 
field $\xi$ defined in $U$ such that $\xi_{p}=\eta$ and for every $q \in U$, hyperplane $\pi_{q}$ spanned by $\left(X_{1}\right)_{q},\left(X_{2}\right)_{q}$ and $\xi_{q}$ is a non-singular local support hyperplane for $M$ at $q$.

Proof. Let $\xi$ be a transversal vector field extending $\eta$, constructed as in Lemma 1 and defined on an open set $V \subset M$. Then for $q \in V$ we consider a function $H_{q}$ defined according to Equation (13). A matrix function $V \ni q \mapsto\left[\Psi_{q}\left(\left(X_{i}\right)_{q},\left(X_{j}\right)_{q}\right)\right]_{i, j=1,2}$ is continuous at $p$, and a matrix $\left[\Psi_{p}\left(\left(X_{i}\right)_{p},\left(X_{j}\right)_{p}\right)\right]_{i, j=1,2}$ is definite, so there is an open set $U \subset V$ containing $p$ such that a matrix $\left[\Psi_{q}\left(\left(X_{i}\right)_{q},\left(X_{j}\right)_{q}\right)\right]_{i, j=1,2}$ is definite for every $q \in U$. Thus, $\Psi_{q}$ is definite in $U$ and, by Proposition 1, each of the hyperplanes $\pi_{q}$ spanned by $\left(X_{1}\right)_{q},\left(X_{2}\right)_{q}$ and $\xi_{q}$ is a non-singular hyperplane supporting a neighborhood of $q$ in $M$. Those hyperplanes obviously vary smoothly with $q$.

Let $M$ be a locally strictly convex surface in $\mathbb{R}^{4}, p \in M$ and $\mathfrak{u}=\left\{X_{1}, X_{2}\right\}$ be a local tangent frame around $p$. It follows that there is a non-singular hyperplane $\pi_{p}$ containing $p$, supporting locally $M$, spanned by $\left(X_{1}\right)_{p},\left(X_{2}\right)_{p}$ and a transversal vector $\eta \in \sigma_{p}$. Applying Propositions 1 and 2 , we can extend $\eta$ to a local transversal vector field $\xi$ such that a bilinear form

$$
G_{\mathfrak{u}}(Y, Z)=\left[X_{1}, X_{2}, D_{Y} Z, \xi\right]
$$

is positive definite (compare Equation (15)). In [2], the form $G_{\mathfrak{u}}$ serves to define another bilinear symmetric form $g_{\xi}$, which turns out to be independent of $\mathfrak{u}$ up to sign:

$$
g_{\xi}(Y, Z)=\frac{G_{\mathfrak{u}}(Y, Z)}{\left(\operatorname{det}_{\mathfrak{u}} G_{\mathfrak{u}}\right)^{1 / 4}} .
$$

A detailed proof is given in [2]. We only notice that $g_{\mathfrak{u}}=g_{\mathfrak{v}}$ for another tangent frame $\mathfrak{v}=\left\{Y_{1}, Y_{2}\right\}$ if the transition matrix between $\mathfrak{u}$ and $\mathfrak{v}$ has a positive determinant. We will show that if a surface $M$ is oriented, we can extend $\xi$ to a global transversal vector field such that the definition of $g_{\xi}$ makes sense.

Proposition 3. Let $M$ be an orientable, locally strictly convex surface in $\mathbb{R}^{4}$. There is an oriented, locally finite atlas $\left\{\left(U_{j}, \phi_{j}\right)\right\}_{j \in J}$ and a family of transversal vector fields $\left\{\xi_{j}\right\}_{j \in J}$, every $\xi_{j}$ defined on $U_{j}$, such that at every point $q \in U_{j}$, the hyperplane spanned by the tangent plane $T_{q} M$ and $\xi_{j}(q)$ supports $M$ locally in a non-singular way. There is also a transversal field $\xi$ on $M$ such that a form $g_{\xi}$ is positive definite.

Proof. We choose an oriented atlas $\left\{\left(W_{i}, \psi_{i}\right)\right\}_{i \in I}$ on $M$. Let $\tilde{\partial}_{i, k}=d \psi_{i}^{-1}\left(e_{k}\right), k=1,2$. According to Propositions 1 and 2, for every point $p \in M$, there exists $i \in I$, an open neighborhood of $p, V_{p} \subset W_{i}$ and a transversal vector field $\xi^{p}$ on $V_{p}$ such that a quadratic form $X \mapsto\left[\tilde{\partial}_{i, 1}, \tilde{\partial}_{i, 2}, D_{X} X, \tilde{\xi}^{p}\right]$ is positive definite. The condition of positivity does not depend on a map $\psi_{i}$ because for another map $\psi_{k}$ such that $W_{i} \cap W_{k} \neq \varnothing$, the Jacobian of $\psi_{i} \circ \psi_{k}^{-1}$ is positive so $\left[\tilde{\partial}_{k, 1}, \tilde{\partial}_{k, 2}, D_{X} X, \tilde{\zeta}^{p}\right]>0$ for a non-zero tangent vector field $X$. Since $M$ is paracompact, there is a locally finite cover $\left\{U_{j}\right\}_{j \in J}$ of $M$ inscribed in $\left\{V_{p}\right\}_{p \in M}$. Thus, for every $j \in J$, there exists $p_{j} \in M$ and $i_{j} \in I$ such that $U_{j} \subset V_{p_{j}} \subset W_{i_{j}}$. Let $\phi_{j}:=\psi_{i_{j}}$ and $\xi_{j}:=\xi^{p_{j}}$ for every $j \in J$. Now we have an oriented and locally finite atlas $\left\{\left(U_{j}, \psi_{j}\right)\right\}_{j \in J}$ on $M$. We also know that, for every $j \in J$, a plane spanned by transversal field $\xi_{j}$, together with the tangent plane, supports $M$ locally in every point of $U_{j}$.

In the next step, we apply a partition of unity $\left\{\lambda_{j}\right\}_{j \in J}$ associated with the cover $\left\{U_{j}\right\}_{j \in J}$ and prove that a transversal vector field $\xi:=\sum_{j \in J} \lambda_{j} \xi_{j}$ satisfies the last assertion of the proposition. Fix $k \in J$. For every $j \in J$ such that $U_{k} \cap U_{j} \neq \varnothing$, we have $\left[\partial_{k, 1}, \partial_{k, 2}, D_{X} X, \xi_{j}\right]>$ 0 in $U_{k} \cap U_{j}$ by a remark in the first part of the proof, where $\partial_{k, l}=d \phi_{k}^{-1}\left(e_{l}\right)(l=1,2)$ and $X$ is a non-zero tangent vector field. Therefore, $\left[\partial_{k, 1}, \partial_{k, 2}, D_{X} X, \sum_{j \in J} \lambda_{j} \xi_{j}\right]>0$ in $U_{k}$. It follows that $g_{\xi}$ is positive definite on $M$.

Proposition 3 leads to the following definition, which is a slight refinement of a notion of a metric field from [2]. It depends on an orientation of $M$ up to sign. 
Definition 2. A transversal vector field $\xi$ such that the bilinear symmetric form $g_{\xi}$ defined by Equation (17) is positive definite is called a metric field.

We remark that Equation (17) always yields a definite bilinear form. In the rest of the paper, we will implicitly assume that the tangent frame $\mathfrak{u}=\left\{X_{1}, X_{2}\right\}$ is chosen so that $g_{\xi}$ is positive definite.

\section{Equiaffine Transversal Bundles}

We recall an important technical theorem from [2].

Theorem 1 ([2], Theorem 3.7). Let $M \subset \mathbb{R}^{4}$ be a locally strictly convex surface and $\xi$ a metric field. Let $\mathfrak{u}=\left\{X_{1}, X_{2}\right\}$ be a local orthonormal tangent frame of $g_{\xi}$ and let $\sigma$ be an arbitrary transversal plane bundle. Then there exists a unique local frame $\left\{\xi_{1}, \xi_{2}\right\}$ of $\sigma$, such that, according to Equation (2), $\left[X_{1}, X_{2}, \xi_{1}, \xi_{2}\right]=1, h^{1}\left(X_{1}, X_{1}\right)=0, \xi+\xi_{1}$ is tangent to $M, h^{2}\left(X_{1}, X_{1}\right)=1$, $h^{2}\left(X_{1}, X_{2}\right)=0$ and $h^{2}\left(X_{2}, X_{2}\right)=1$.

We remark that the condition that $\xi+\xi_{1}$ is tangent to $M$ implies that $g_{\xi}=g_{-} \xi_{1}$. We also note that the proof of the above theorem implies that the tangent frame $\mathfrak{u}$ does not need to be positively oriented.

For a given transversal bundle $\sigma$ and a metric field $\xi$, a metric $g^{\perp}$ on $\sigma$ is given locally in the following way. We take a local $g_{\xi}$-orthonormal tangent frame $\mathfrak{u}=\left\{X_{1}, X_{2}\right\}$ and the corresponding local transversal frame $\left\{\xi_{1}, \xi_{2}\right\}$ obtained by Theorem 1 . We assume that $h^{1}$ does not vanish (we will recall below that it is equivalent to the inexistence of inflection points). Then we define a metric $g_{\mathfrak{u}}^{\perp}$ using the local basis $\left\{\xi_{1}, \xi_{2}\right\}$ :

$$
\begin{aligned}
& g_{\mathfrak{u}}^{\perp}\left(\xi_{1}, \xi_{1}\right)=1, \\
& g_{\mathfrak{u}}^{\perp}\left(\xi_{1}, \xi_{2}\right)=-\frac{1}{2} h_{1}\left(X_{2}, X_{2}\right), \\
& g_{\mathfrak{u}}^{\perp}\left(\xi_{2}, \xi_{2}\right)=4 h_{1}\left(X_{1}, X_{2}\right)^{2}+\frac{5}{4} h_{1}\left(X_{2}, X_{2}\right)^{2} .
\end{aligned}
$$

This metric does not depend on $\mathfrak{u}$ (see [2]), so we will denote it by $g^{\perp}$.

For a given metric field $\xi$, a specific transversal bundle $\sigma$ is proposed in [2]. It is suitable for locally strictly convex surfaces, which will be explained later. It is equiaffine in the sense that $\nabla \omega_{g}=0$, where $\nabla$ is the affine connection induced by $\sigma$, and $\omega_{g}$ is the volume element associated with the metric $g=g_{\xi}$. It is shown in [2] that $\sigma$ is equiaffine if and only if

$$
\begin{aligned}
& (\nabla g)\left(X_{1}, X_{1}, X_{1}\right)+(\nabla g)\left(X_{1}, X_{2}, X_{2}\right)=0 \\
& (\nabla g)\left(X_{2}, X_{1}, X_{1}\right)+(\nabla g)\left(X_{2}, X_{2}, X_{2}\right)=0
\end{aligned}
$$

where $\left\{X_{1}, X_{2}\right\}$ is an orthonormal tangent frame with respect to $g$. An important assumption that allows the construction of $\sigma$ in a neighborhood of $p \in M$ is that $p$ is not an inflection point. An equivalent condition is given in [2]: $p$ is an inflection if and only if $h^{1}\left(X_{1}, X_{2}\right)=h^{1}\left(X_{2}, X_{2}\right)=0$ for an orthonormal tangent frame $\mathfrak{u}=\left\{X_{1}, X_{2}\right\}$ and the associated transversal frame $\left\{\xi_{1}, \xi_{2}\right\}$ with respect to which $h^{1}$ and $h^{2}$ are determined. We note that if a surface is contained in a three-dimensional affine subspace of $\mathbb{R}^{4}$, then it consists of only inflection points. The uniqueness of the construction is possible under the following symmetry conditions.

Definition 3. Let $\mathfrak{u}=\left\{X_{1}, X_{2}\right\}$ be a g-orthonormal tangent frame. An equiaffine plane bundle $\sigma$ is symmetric, if

$$
\begin{aligned}
& (\nabla g)\left(X_{2}, X_{1}, X_{1}\right)-(\nabla g)\left(X_{1}, X_{2}, X_{1}\right)=0 \\
& (\nabla g)\left(X_{1}, X_{2}, X_{2}\right)-(\nabla g)\left(X_{2}, X_{1}, X_{2}\right)=0
\end{aligned}
$$


and antisymmetric, if

$$
\begin{aligned}
& (\nabla g)\left(X_{2}, X_{1}, X_{1}\right)+(\nabla g)\left(X_{1}, X_{2}, X_{1}\right)=0 \\
& (\nabla g)\left(X_{1}, X_{2}, X_{2}\right)+(\nabla g)\left(X_{2}, X_{1}, X_{2}\right)=0 .
\end{aligned}
$$

The following statement comes from [2].

Theorem 2 ([2], Theorems 5.4 and 5.5). Let $M \subset \mathbb{R}^{4}$ be a locally strictly convex surface and $\xi$ be a metric field. If $p \in M$ is not an inflection, then there exists a unique antisymmetric equiaffine plane bundle and a unique symmetric equiaffine plane bundle defined on a neighborhood of $p$.

\section{Locally Strictly Convex Surfaces Contained in Hyperquadrics}

In this section, we study locally strictly convex surfaces embedded in hyperquadrics in $\mathbb{R}^{4}$. We use two specific transversal bundles, which are constructed in [2]. In fact, the bundles coincide in the discussed case. The authors of [2] claim that their bundle is especially well designed for surfaces embedded in hyperquadrics because a canonically determined Blaschke normal of hyperquadric is contained in this bundle, which is not true for Nomizu-Vrancken bundle (see [NV], also [2], Remark 7.4). That statement is a part of Theorem 7.3 in [2]. We prove that the assertion of the theorem is true; however, the original proof contains an essential gap. We restate that part of their conclusions, and then we complete a proof of the statement of the theorem. We also assume that there are no inflection points.

Let $M$ be a surface embedded in a locally strictly convex hypersurface $N \subset \mathbb{R}^{4}$. We recall that such a surface is locally strictly convex itself. Let $\mathcal{Y}$ be a transversal field on $N$. It induces an affine connection $\tilde{\nabla}$ on $N$ and the second fundamental form $h$ by the following formula of Gauss:

$$
D_{X} Z=\tilde{\nabla}_{X} Z+h(X, Z) \mathcal{Y}
$$

where $X, Z$ are vector fields tangent to $N$. In the case of locally strictly convex hypersurfaces, $h$ is non-degenerate, and it can be regarded as a metric on $N$. We recall a notion of a Blaschke normal (see (NS) for details). Blaschke normal is a transversal field $\mathcal{Y}$, which is determined up to sign by two properties:

(1) the connection $\tilde{\nabla}$ in Equation (21) is equiaffine, which is equivalent to the fact that $D_{X} \mathcal{Y}$ is tangent to $N$ for every tangent vector field $X$;

(2) the volume element $\omega_{h}$ associated with metric $h$ is equal to a volume element $\theta\left(X_{1}, X_{2}, X_{3}\right):=\left[X_{1}, X_{2}, X_{3}, \mathcal{Y}\right]$ induced by $\mathcal{Y}$ on $N$.

We also recall a fact (see [3]) that $N$ is a hyperquadric if and only if the cubic form $C=\tilde{\nabla} h$ vanishes identically.

In the following proposition, we correct a part of the proof of Theorem 7.3 from [2]. It will be useful to give a complete proof of that result. We recall that a hypersurface is non-degenerate if its second fundamental form is non-degenerate.

Proposition 4. Let $\xi$ be a metric field on a locally strictly convex connected surface M embedded in a non-degenerate hyperquadric $N \subset \mathbb{R}^{4}$. We assume that $\xi$ is tangent to $N$, and there are no inflection points on $M$. Let $X_{1}, X_{2}$ be a $g_{\xi}$-orthonormal frame on $M$ and $f>0$ a function such that $\left[X_{1}, X_{2},-\xi, f \mathcal{Y}\right]=1$ on $M$, where $\mathcal{Y}$ is a Blaschke normal on $N$. Then $\mathcal{Y}$ restricted to $M$ belongs to the equiaffine antisymmetric bundle if and only if $f$ is constant.

Proof. Since $\xi$ is tangent to $N$ and $\mathcal{Y}$ is transversal to it, then a bundle $\sigma$ on $M$ is generated by $\xi$, and $\mathcal{Y}$ is a plane bundle. It is obviously transversal to $M$. By Theorem 1 , there exists a transversal frame $\left\{\xi_{1}, \xi_{2}\right\}$ in $\sigma$ such that $\xi_{1}+\xi$ is tangent to $M,\left[X_{1}, X_{2}, \xi_{1}, \xi_{2}\right]=1$, $h^{1}\left(X_{1}, X_{1}\right)=0$ and $h^{2}\left(X_{i}, X_{j}\right)=\delta_{i j}$. Our assumption and the proof of the theorem imply that the transversal frame is defined on $M$. Since $\left[X_{1}, X_{2}, \xi_{1}, f \mathcal{Y}\right]=\left[X_{1}, X_{2}, \xi_{1}, \xi_{2}\right]=1$, 
there exists a function $\lambda_{1}$ such that $\xi_{2}=\lambda_{1} \xi_{1}+f \mathcal{Y}$. Denoting by $\nabla$, as usual, the connection induced by $\sigma$ on $M$, we define functions $a_{1}$ to $a_{8}$ such that

$$
\begin{array}{ll}
\nabla_{X_{1}} X_{1}=a_{1} X_{1}+a_{2} X_{2}, & \nabla_{X_{1}} X_{2}=a_{3} X_{1}+a_{4} X_{2} \\
\nabla_{X_{2}} X_{1}=a_{5} X_{1}+a_{6} X_{2}, & \nabla_{X_{2}} X_{2}=a_{7} X_{1}+a_{8} X_{2} .
\end{array}
$$

Next, we make some preparatory computations, using the above facts and notations.

$$
\begin{aligned}
& D_{X_{1}} X_{1}=a_{1} X_{1}+a_{2} X_{2}+\xi_{2} \\
& =a_{1} X_{1}+a_{2} X_{2}+\lambda_{1} \xi_{1}+f \mathcal{Y}, \\
& D_{X_{1}} X_{2}=a_{3} X_{1}+a_{4} X_{2}+h^{1}\left(X_{1}, X_{2}\right) \xi_{1}, \\
& D_{X_{2}} X_{1}=a_{5} X_{1}+a_{6} X_{2}+h^{1}\left(X_{1}, X_{2}\right) \xi_{1}, \\
& D_{X_{2}} X_{2}=a_{7} X_{1}+a_{8} X_{2}+h^{1}\left(X_{2}, X_{2}\right) \xi_{1}+\lambda_{1} \xi_{1}+f \mathcal{Y}, \\
& D_{X_{1}} \xi_{1}=-S_{1} X_{1}+\tau_{1}^{1}\left(X_{1}\right) \xi_{1}+\tau_{1}^{2}\left(X_{1}\right) \underbrace{\left(\lambda_{1} \xi_{1}+f \mathcal{Y}\right)}_{\xi_{2}}, \\
& D_{X_{2}} \xi_{1}=-S_{1} X_{2}+\tau_{1}^{1}\left(X_{2}\right) \xi_{1}+\tau_{1}^{2}\left(X_{2}\right)\left(\lambda_{1} \xi_{1}+f \mathcal{Y}\right) .
\end{aligned}
$$
deduce:

Taking Equation (21) into consideration and remembering that $\xi_{1}$ is tangent to $N$, we

$$
\begin{aligned}
& h\left(X_{1}, X_{1}\right)=f, \quad h\left(X_{2}, X_{2}\right)=f, \quad h\left(X_{1}, X_{2}\right)=0, \\
& h\left(X_{1}, \xi_{1}\right)=\tau_{1}^{2}\left(X_{1}\right) f, \quad h\left(X_{2}, \xi_{1}\right)=\tau_{1}^{2}\left(X_{2}\right) f .
\end{aligned}
$$

In the next step, we apply the assumption that the cubic form on $N$ vanishes. We also use Equations (23).

$$
\begin{aligned}
0 & =C\left(X_{1}, X_{1}, X_{1}\right)=X_{1}(f)-2 h\left(a_{1} X_{1}+a_{2} X_{2}+\lambda_{1} \xi_{1}\right) \\
& =X_{1}(f)-2 a_{1} f-2 \lambda_{1} \tau_{1}^{2}\left(X_{1}\right) f, \\
0 & =C\left(X_{1}, X_{1}, X_{2}\right)=-h\left(\tilde{\nabla}_{X_{1}} X_{1}, X_{2}\right)-h\left(X_{1}, \tilde{\nabla}_{X_{1}} X_{2}\right) \\
& =-h\left(a_{1} X_{1}+a_{2} X_{2}+\lambda_{1} \xi_{1}, X_{2}\right)-h\left(a_{3} X_{1}+a_{4} X_{2}+h^{1}\left(X_{1}, X_{2}\right) \xi_{1}\right) \\
& =-a_{2} f-\lambda_{1} \tau_{1}^{2}\left(X_{2}\right) f-a_{3} f-h^{1}\left(X_{1}, X_{2}\right) \tau_{1}^{2}\left(X_{1}\right) f, \\
0 & =C\left(X_{1}, X_{2}, X_{2}\right)=X_{1}(f)-2 h\left(\tilde{\nabla}_{X_{1}} X_{2}, X_{2}\right. \\
& =X_{1}(f)-2 a_{4} f-2 h^{1}\left(X_{1}, X_{2}\right) \tau_{1}^{2}\left(X_{2}\right) f, \\
0 & =C\left(X_{2}, X_{1}, X_{1}\right)=X_{2}(f)-2 h\left(a_{5} X_{1}+a_{6} X_{2}+h^{1}\left(X_{1}, X_{2}\right) \xi_{1}, X_{1}\right) \\
& =X_{2}(f)-2 a_{5} f-h^{1}\left(X_{1}, X_{2}\right) \tau_{1}^{2}\left(X_{1}\right) f \\
0 & =C\left(X_{2}, X_{1}, X_{2}\right)=-h\left(\tilde{\nabla}_{X_{2}} X_{1}, X_{2}\right)-h\left(X_{1}, \tilde{\nabla}_{X_{2}} X_{2}\right) \\
& =-h\left(a_{5} X_{1}+a_{6} X_{2}+h^{1}\left(X_{1}, X_{2}\right) \xi_{1}, X_{2}\right)-h\left(a_{7} X_{1}+a_{8} X_{2}+h^{!}\left(X_{2}, X_{2}\right) \xi_{1}+\lambda_{1} \xi_{1}\right) \\
& =-a_{6} f-h^{1}\left(X_{1}, X_{2}\right) \tau_{1}^{2}\left(X_{2}\right) f-a_{7} f-\left(h^{1}\left(X_{2}, X_{2}\right)+\lambda_{1}\right) \tau_{1}^{2}\left(X_{1}\right) f, \\
0 & =C\left(X_{2}, X_{2}, X_{2}\right)=X_{2}(f)-2 h\left(a_{7} X_{1}+a_{8} X_{2}+h^{1}\left(X_{2}, X_{2}\right) \xi_{1}+\lambda_{1} \xi_{1}, X_{2}\right) \\
& =X_{2}(f)-2 a_{8} f-2\left(h^{1}\left(X_{2}, X_{2}\right)+\lambda_{1}\right) \tau_{1}^{2}\left(X_{2}\right) f .
\end{aligned}
$$

Applying Remark 5.6 from [2], we observe that the antisymmetric transversal bundle is generated by vector fields $\bar{\xi}_{1}, \bar{\xi}_{2}$ of the form

$$
\begin{aligned}
& \bar{\xi}_{1}=\xi_{1}-a X_{1}-b X_{2} \\
& \bar{\xi}_{2}=\xi_{2}-c X_{1}-d X_{2}
\end{aligned}
$$


where

$$
\begin{aligned}
& a=\frac{-2\left(a_{2}+a_{3}+a_{5}-a_{8}\right) h^{1}\left(X_{1}, X_{2}\right)-\left(a_{4}+a_{6}+a_{7}-a_{1}\right) h^{1}\left(X_{2}, X_{2}\right)}{4\left(h^{1}\left(X_{1}, X_{2}\right)\right)^{2}+\left(h^{1}\left(X_{2}, X_{2}\right)\right)^{2}} \\
& b=\frac{-2\left(a_{4}+a_{6}+a_{7}-a_{1}\right) h^{1}\left(X_{1}, X_{2}\right)-\left(a_{2}+a_{3}+a_{5}-a_{8}\right) h^{1}\left(X_{2}, X_{2}\right)}{4\left(h^{1}\left(X_{1}, X_{2}\right)\right)^{2}+\left(h^{1}\left(X_{2}, X_{2}\right)\right)^{2}} \\
& c=-\left(a_{1}+a_{4}+b h^{1}\left(X_{1}, X_{2}\right)\right) \\
& d=-\left(a_{5}+a_{8}+a h^{1}\left(X_{1}, X_{2}\right)+b h^{1}\left(X_{2}, X_{2}\right)\right) .
\end{aligned}
$$

By Equations (24), (26) and (28), we have

$$
a_{1}-a_{4}-a_{6}-a_{7}=2 h^{1}\left(X_{1}, X_{2}\right) \tau_{1}^{2}\left(X_{2}\right)+h^{1}\left(X_{2}, X_{2}\right) \tau_{1}^{2}\left(X_{1}\right)
$$

and from Equations (25), (27) and (29) we get

$$
a_{8}-a_{5}-a_{2}-a_{3}=2 h^{1}\left(X_{1}, X_{2}\right) \tau_{1}^{2}\left(X_{1}\right)-h^{1}\left(X_{2}, X_{2}\right) \tau_{1}^{2}\left(X_{2}\right) .
$$

Hence, after straightforward computations using Equations (32) and (33), we obtain $a=$ $\tau_{1}^{2}\left(X_{1}\right)$ and $b=\tau_{1}^{2}\left(X_{2}\right)$. Applying additionally Equations (24), (26) and (34), once again, we get

$$
c=\lambda_{1} \tau_{1}^{2}\left(X_{1}\right)-X_{1}(\log f)
$$

and from Equations (27), (29) and (35), we have

$$
d=\lambda_{1} \tau_{1}^{2}\left(X_{2}\right)-X_{2}(\log f) .
$$

Finally, after substituting the above formulas for $a, b, c$ and $d$ into Equations (30) and (31) we obtain:

$$
\begin{aligned}
\bar{\xi}_{1} & =\xi_{1}-\tau_{1}^{2}\left(X_{1}\right) X_{1}-\tau_{1}^{2}\left(X_{2}\right) X_{2}, \\
\bar{\xi}_{2} & =\xi_{2}-\lambda_{1} \tau_{1}^{2}\left(X_{1}\right) X_{1}+X_{1}(\log f) X_{1}-\lambda_{1} \tau_{1}^{2}\left(X_{2}\right) X_{2}+X_{2}(\log f) X_{2} \\
& =\lambda_{1} \xi_{1}+f \mathcal{Y}+\lambda_{1}\left(\bar{\xi}_{1}-\xi_{1}\right)+X_{1}(\log f) X_{1}+X_{2}(\log f) X_{2},
\end{aligned}
$$

hence

$$
f \mathcal{Y}=\bar{\xi}_{2}-\lambda_{1} \bar{\xi}_{1}-X_{1}(\log f) X_{1}-X_{2}(\log f) X_{2}
$$

so $\mathcal{Y}$ belongs to the antisymmetric transversal bundle if and only if $X_{1}(\log f)=0$ and $X_{2}(\log f)=0$, which completes the proof because $M$ is connected.

Remark 2. Proposition 4 is also true for the symmetric equiaffine bundle. We omit the calculations because they are analogous to those in the antisymmetric case.

Let $\mathfrak{G}$ be the Blaschke metric on a locally strictly convex hyperquadric $N$ containing $M$ (see [4]). It is shown in [2] that for every transversal bundle $\sigma$ on $M$, there is a transversal (that means $\sigma$-valued) field $\xi$ on $M$ such that $\mathfrak{G}$ restricted to $M$ coincides with $g_{\xi}$. We investigate some details of the construction.

Lemma 2. For any point $p \in M$, there is a metric field $\xi$ defined in a connected neighborhood of $p$ such that it is tangent to $N$ and $g_{\xi}$ coincides with the Blaschke metric $\mathfrak{G}$ on $N$ restricted to M. Moreover, for any $g_{\xi}$-orthonormal transversal frame $\left\{\tilde{X}_{1}, \tilde{X}_{2}\right\}$ and for the Blaschke normal $\mathcal{Y}$, $\left[\tilde{X}_{1}, \tilde{X}_{2},-\xi, \mathcal{Y}\right]=1$. 
Proof. Let $p \in M$ and $U$ be a connected, open neighborhood of $p$. The proof will be carried on in $U$. Let $\mathfrak{u}^{\prime}=\left\{Y_{1}, Y_{2}, Y_{3}\right\}$ be a local tangent frame on $N$ and $Z, W$ be arbitrary tangent vector fields on $N$ such that they are also defined in $U$. Then

$$
H_{\mathfrak{u}^{\prime}}(Z, W):=\left[Y_{1}, Y_{2}, Y_{3}, D_{Z} W\right]
$$

is a bilinear symmetric form, which is non-degenerate because $N$ is locally strictly convex. A bilinear symmetric form

$$
\mathfrak{G}(Z, W):=\frac{H_{\mathfrak{u}^{\prime}}(Z, W)}{\left(\operatorname{det}_{\mathfrak{u}^{\prime}} H_{\mathfrak{u}^{\prime}}\right)^{\frac{1}{5}}}
$$

does not depend on $\mathfrak{u}^{\prime}$, and it is called the Blaschke metric.

Let $\mathfrak{u}=\left\{X_{1}, X_{2}\right\}$ be a local tangent frame on $M$. Let $X_{3}$ be such a local tangent vector field on $N$ that $X_{1}, X_{2}$ and $X_{3}$ form a basis of the tangent space to $N$ at points of $M$. Denote this frame by $\mathfrak{u}^{\prime}$. It is obvious that $X_{3}$ is transversal to $M$. Take a function

$$
\lambda:=\frac{\left(\operatorname{det}_{\mathfrak{u}^{\prime}} H_{\mathfrak{u}^{\prime}}\right)^{\frac{1}{5}}}{\left(\operatorname{det}_{\mathfrak{u}} G_{\mathfrak{u}}\right)^{\frac{1}{4}}} .
$$

It is well-defined on $M$ because we identify $H_{\mathfrak{u}^{\prime}}\left(X_{3}, X_{i}\right)$ with $H_{\mathfrak{u}^{\prime}}\left(X_{i}, X_{3}\right)$ for $i=1,2$. We could also extend $X_{1}$ and $X_{2}$ on $N$ locally with the same effect. Then we obtain a metric field $\xi=-\lambda^{-2} X_{3}$ such that

$$
g_{\xi}=\mathfrak{G}_{\mid M}
$$

locally (see [2]). In the next step, we take a $g_{\xi}$ local orthonormal frame $\tilde{\mathfrak{u}}=\left\{\tilde{X}_{1}, \tilde{X}_{2}\right\}$ on $M$. Then $G_{\tilde{u}}\left(\tilde{X}_{1}, \tilde{X}_{2}\right)=0$ and $G_{\tilde{u}}\left(\tilde{X}_{i}, \tilde{X}_{i}\right)=\left(\operatorname{det}_{\tilde{u}} G_{\tilde{u}}\right)^{\frac{1}{4}}$ for $i=1,2$. Consequently, $\operatorname{det}_{\tilde{u}} G_{\tilde{u}}=G_{\tilde{u}}\left(\tilde{X}_{1}, \tilde{X}_{1}\right) G_{\tilde{\mathfrak{u}}}\left(\tilde{X}_{2}, \tilde{X}_{2}\right)$, so $\operatorname{det}_{\tilde{\mathfrak{u}}} G_{\tilde{\mathfrak{u}}}=\left(\operatorname{det}_{\tilde{u}} G_{\tilde{\mathfrak{u}}}\right)^{\frac{1}{2}}$, and finally $\operatorname{det}_{\tilde{\mathfrak{u}}} G_{\tilde{\mathfrak{u}}}=1$.

Let $\tilde{X}_{3}:=-\tilde{\xi}=\lambda^{-2} X_{3}$. Using the notation $\tilde{\mathfrak{u}}^{\prime}=\left\{\tilde{X}_{1}, \tilde{X}_{2}, \tilde{X}_{3}\right\}$ and extending $\lambda$ on an open set in $N$, we can compute $H_{\tilde{u}^{\prime}}$ locally on $M$. The result clearly does not depend on a particular extension of $\lambda$ because the term containing $X_{3}(\lambda) X_{3}$ is canceled in $H_{\mathfrak{u}^{\prime}}\left(\tilde{X}_{3}, \tilde{X}_{3}\right)$. We repeat a construction of a metric field satisfying a condition analogous to Equation (36), starting with $\tilde{\mathfrak{u}}$ and $\tilde{\mathfrak{u}}^{\prime}$. Thus, we define a function

$$
\tilde{\lambda}:=\frac{\left(\operatorname{det}_{\tilde{\mathfrak{u}}^{\prime}} H_{\tilde{\mathfrak{u}}^{\prime}}\right)^{\frac{1}{5}}}{\left(\operatorname{det}_{\tilde{\mathfrak{u}}} G_{\tilde{\mathfrak{u}}}\right)^{\frac{1}{4}}}=\left(\operatorname{det}_{\tilde{\mathfrak{u}}^{\prime}} H_{\tilde{\mathfrak{u}}^{\prime}}\right)^{\frac{1}{5}}
$$

and obtain a metric field $\frac{\xi}{\tilde{\lambda}^{2}}$ satisfying

$$
g_{\frac{\xi}{\bar{\lambda}^{2}}}=\mathfrak{G}_{\mid M}=g_{\xi} .
$$

Since $g_{\frac{\xi}{\tilde{\lambda}^{2}}}=\frac{1}{|\tilde{\lambda}|} g_{\tilde{\xi}}$, we have $|\tilde{\lambda}|=1$ and

$$
\left|\operatorname{det}_{\tilde{\mathfrak{u}}^{\prime}} H_{\tilde{\mathfrak{u}}^{\prime}}\right|=1 .
$$

Let $\sigma$ be a local transversal bundle on $M$ generated by vector fields $\xi$ and $\mathcal{Y}$, where $\mathcal{Y}$ is a Blaschke normal on $N$. Since $\left\{\tilde{X}_{1}, \tilde{X}_{2}\right\}$ is a $g_{\xi}$-orthonormal tangent frame, we apply Theorem 1 . According to it, there exists a transversal frame $\left\{\xi_{1}, \xi_{2}\right\}$ in $\sigma$ such that $\xi+\xi_{1}$ is tangent to $M,\left[\tilde{X}_{1}, \tilde{X}_{2}, \xi_{1}, \xi_{2}\right]=1, h^{1}\left(\tilde{X}_{1}, \tilde{X}_{1}\right)=0$ and $h^{2}\left(\tilde{X}_{i}, \tilde{X}_{j}\right)=\delta_{i j}, i, j=1,2$. It implies that $\xi_{2}$ is transversal to $N$. From the procedure of constructing a Blaschke normal (NS), we know that we can start it with $\xi_{2}$ as a transversal field to $N$ and then $\mathcal{Y}=\left|\operatorname{det}_{\tilde{\mathfrak{u}}^{\prime}} H_{\tilde{\mathfrak{u}}^{\prime}}\right| \xi_{2}+Z=\xi_{2}+Z$, where $Z$ is tangent to $N$. The construction is done at points of $M$. However, $\xi_{2}$ is a linear combination of $\xi_{1}$ and $\mathcal{Y}$, so there is a function $\lambda_{1}$ such that $\mathcal{Y}=\xi_{2}+\lambda_{1} \xi$. Hence, $\left[\tilde{X}_{1}, \tilde{X}_{2},-\xi, \mathcal{Y}\right]=\left[\tilde{X}_{1}, \tilde{X}_{2},-\xi_{,} \xi_{2}\right]=\left[\tilde{X}_{1}, \tilde{X}_{2}, \xi_{1}, \xi_{2}\right]=1$, which completes the proof. 
The above lemma together with Proposition 4 prove the following theorem from [2]:

Theorem 3. Let $M \subset \mathbb{R}^{4}$ be a locally strictly convex surface immersed in a hyperquadric $N$. Then the affine normal field to $N$ belongs to both the antisymmetric and symmetric equiaffine plane bundles of $M$, with respect to the Blaschke metric $\mathfrak{G}$ restricted to $M$.

Remark 3. In [2], the proof of the above theorem apparently presupposes that the function $f$ appearing in Proposition 4 is equal to 1, but no verification of this fact is presented.

In the remainder of this section, we present three examples of surfaces contained in strictly convex hyperquadrics. We endow each example in a parametrization that is suitable for presenting the geometric properties of our interest. Only the first one will contain detailed computations.

Example 1 (Clifford torus). Let $y(u, v, w)=\sqrt{2} \sqrt[8]{2}(\cos u \sin w, \sin u \sin w, \cos v \cos w, \sin v$ $\cos w)$ be a parametrization of a three-dimensional sphere $\mathbb{S}^{3}$ of a radius $2 \sqrt[4]{2}$. Consider a Clifford torus $M_{1}=\mathbb{S}^{1} \times \mathbb{S}^{1}$ contained in $\mathbb{S}^{3}$ parametrized by

$$
x(u, v)=y(u, v, \pi / 4)=\sqrt[8]{2}(\cos u, \sin u, \cos v, \sin v)
$$

where both circles also have the radius $2 \sqrt[4]{2}$. First, we determine a frame $\mathfrak{u}^{\prime}=\left\{X_{1}, X_{2}, X_{3}\right\}$ tangent to $\mathbb{S}^{3}$ but defined in $M_{1}$ :

$$
\begin{aligned}
& X_{1}=x_{u}=\sqrt[8]{2}(-\sin u, \cos u, 0,0) \\
& X_{2}=x_{v}=\sqrt[8]{2}(0,0, \sin v, \cos v) \\
& X_{3}=y_{w}(u, v, \pi / 4)=\sqrt[8]{2}(\cos u, \sin u,-\cos v,-\sin v) .
\end{aligned}
$$

Next, we compute $H_{\mathfrak{u}^{\prime}}\left(X_{i}, X_{j}\right)$ for all $i, j$, taking into account that $D_{X_{1}} X_{1}=x_{u u}, D_{X_{2}} X_{2}=$ $x_{v v}, D_{X_{1}} X_{2}=D_{X_{2}} X_{1}=x_{u v}=0$ and $D_{X_{3}} X_{3}=y_{w w}(u, v, \pi / 4)=\sqrt[8]{2}(-\cos u,-\sin u$, $-\cos v,-\sin v)$. We get

$$
\operatorname{det}_{\mathfrak{u}^{\prime}} H_{\mathfrak{u}^{\prime}}=\operatorname{det}\left[\begin{array}{ccc}
\sqrt{2} & 0 & 0 \\
0 & \sqrt{2} & 0 \\
0 & 0 & 2 \sqrt{2}
\end{array}\right]=4 \sqrt{2} \text {. }
$$

We immediately get $\operatorname{det}_{\mathfrak{u}} G_{\mathfrak{u}}=2$, where $\mathfrak{u}=\left\{X_{1}, X_{2}\right\}$ and $G_{\mathfrak{u}}$ is computed with respect to a metric field $-X_{3}$. According to the proof of Lemma 2 , for $\lambda=\sqrt[4]{2}$, we obtain another metric field $\xi=-\lambda^{-2} X_{3}$ such that $g_{\xi}$ coincides with the Blaschke metric on $\mathbb{S}^{3}$ induced on $M_{1}$. Since $g_{\xi}=\lambda^{-1} g_{-X_{3}}, \mathfrak{u}$ is a $g_{\xi}$-orthonormal frame that can be easily seen. For $\xi_{1}=-\xi$ and $\xi_{2}=\sqrt[8]{2}(-\cos u,-\sin u, 0,0)$, we also get $\left[X_{1}, X_{2}, \xi_{1}, \xi_{2}\right]=1$. Then

$$
D_{X_{1}} X_{1}=\xi_{2}, \quad D_{X_{1}} X_{2}=0, \quad D_{X_{2}} X_{2}=-\sqrt{2} \xi_{1}+\xi_{2}
$$

which implies that $\left\{\xi_{1}, \xi_{2}\right\}$ is the transversal frame associated with $\mathfrak{u}$ according to Theorem 1 in a transversal bundle $\sigma$ generated by $\xi_{1}$ and $\xi_{2}$. In particular, $h^{1}\left(X_{1}, X_{2}\right)=0$ and $h^{1}\left(X_{2}, X_{2}\right)=$ $-\sqrt{2}$. From Equation (37), we also deduce that $\nabla_{X_{i}} X_{j}=0$ for all $i, j=1,2$. Hence, $\nabla g_{\xi}=0$, and by Equations (18)-(20), we conclude that $\sigma$ is the equiaffine transversal bundle that is both symmetric and antisymmetric. We can also see that all $D_{X_{i}} \xi_{j}$ are tangent to $M_{1}$. As a consequence, $K^{1}=K^{2}=0$ identically. After straightforward computations, we also obtain $\nabla^{\perp} g_{\xi}^{\perp}=0$.

Example 2 (The product of an ellipse and a hyperbola). Let $y(u, v, w)=(\cos u \sinh w, \sin u$ $\left.\sinh w, \sinh \left(\frac{v}{\sqrt{2}}\right) \cosh w, \cosh \left(\frac{v}{\sqrt{2}}\right) \cosh w\right)$ be a parametrization of a one sheet $N_{2}$ of a hyperboloid $y_{1}^{2}+y_{2}^{2}+y_{3}^{2}-y_{4}^{2}=-1$, which is strictly convex. We consider a surface $M_{2}$ parametrized by $x(u, v)=y\left(u, v, w_{0}\right)$, where $w_{0}=\log (1+\sqrt{2})$, so $\sinh w_{0}=1$ and $\cosh w_{0}=\sqrt{2}$. Therefore, 


$$
x(u, v)=\left(\cos u, \sin u, \sqrt{2} \sinh \left(\frac{v}{\sqrt{2}}\right), \sqrt{2} \cosh \left(\frac{v}{\sqrt{2}}\right) .\right.
$$

We take vector fields $X_{1}=x_{u}, X_{2}=x_{v}$ and $X_{3}=y_{w}\left(u, v, w_{0}\right)$ on $M_{2}$, following the pattern of Example 1. We also need a covariant derivative $D_{X_{3}} X_{3}$ restricted to $M_{2}$, so $D_{X_{3}} X_{3}=$ $y_{w w}\left(u, v, w_{0}\right)$. After straightforward calculations, we deduce that $\xi=-X_{3}$ is a suitable metric field such that $g_{\xi}$ coincides with the Blaschke metric restricted to $M_{2}$. We also can check that $\left\{X_{1}, X_{2}\right\}$ is a $g_{\xi}$-orthonormal frame. For $\xi_{1}=-\xi$ and $\xi_{2}=D_{X_{1}} X_{1},\left\{\xi_{1}, \xi_{2}\right\}$ is the associated transversal frame in a bundle $\sigma$, which it generates. A transversal bundle $\sigma$ is the equiaffine symmetric and antisymmetric one. The cubic forms $K^{1}$ and $K^{2}$ with respect to $\sigma$ vanish and $\nabla^{\perp} g_{\xi}^{\perp}=0$.

Example 3 (The product of an ellipse and a parabola). Let $y(u, v, w)=\frac{1}{\sqrt[4]{2}}(w \cos u, w \sin u, v$, $\left.v^{2}+w^{2}\right)$ be a parametrization of a paraboloid $N_{3}: y_{4}=\sqrt[4]{2}\left(y_{1}^{2}+y_{2}^{2}+y_{3}^{2}\right)$. We consider a surface $x(u, v)=y(u, v, 1)=\frac{1}{\sqrt[4]{2}}\left(\cos u, \sin u, v, v^{2}+1\right)$, which is contained in $N_{3}$. We take $\mathfrak{u}=\left\{X_{1}, X_{2}\right\}$ as a tangent frame on the surface for $X_{1}=\frac{1}{\sqrt[4]{2}}(-\sin u, \cos u, 0,0)$ and $X_{2}=\frac{1}{\sqrt[4]{2}}(0,0,1,2 v)$. As a metric field, we choose $\xi=-y_{w}(u, v, 1)=-\frac{1}{\sqrt[4]{2}}(\cos u, \sin u, 0,2)$. It is easy to verify, like in previous examples, that $g_{\xi}$ coincides with the Blaschke metric on $\mathrm{N}_{3}$ restricted to the surface. It also turns out that $\mathfrak{u}$ is a $g_{\xi}$-orthonormal frame. Let $\sigma$ be a transversal bundle generated by vector fields $\xi_{1}=-\xi$ and $\xi_{2}=x_{u u}=\frac{1}{\sqrt[4]{2}}(-\cos u,-\sin u, 0,0)$. Then, after straightforward computations, we can see that $\left\{\xi_{1}, \xi_{2}\right\}$ is the transversal frame associated with $\mathfrak{u}$ in $\sigma$. Moreover, $\sigma$ is the equiaffine symmetric and antisymmetric transversal bundle such that the induced normal connection has the properties $\nabla^{\perp} g_{\xi} \frac{\perp}{\xi}=0$ and $K^{1}=K^{2}=0$.

\section{Classification of Parallel Surfaces Contained in Locally Strictly Convex Hyperquadrics}

The main aim of this paper is to characterize surfaces without inflection points contained in locally strictly convex hyperquadrics. We recall that a surface contained in a locally strictly convex hypersurface in $\mathbb{R}^{4}$ is always locally strictly convex itself. We choose a metric field satisfying Equation (36). We assume additionally that the cubic form $K$ of a surface vanishes and $\nabla^{\perp} g_{\xi} \frac{\perp}{\xi}=0$. Both connections, $\nabla$ and $\nabla^{\perp}$, are induced by the equiaffine transversal bundle that is constructed in [2]. We can use either a symmetric or antisymmetric bundle because they are identical in the case of this study. We note that the assumptions given above are invariant under a change of a given tangent frame and a transversal frame.

Let $\xi$ be a metric field on a surface $M$ embedded in a locally strictly convex hyperquadric $N$ of $\mathbb{R}^{4}$ such that $g=g_{\xi}$ coincides with the Blaschke metric $\mathfrak{G}$ on $N$ restricted to $M$. Let $\sigma$ be the antisymmetric equiaffine plane bundle. Our considerations in this section will be local, so $M$ will denote an open and connected subset of the surface. Let $\left\{X_{1}, X_{2}\right\}$ be an orthonormal tangent frame on $M$ and $\left\{\xi_{1}, \xi_{2}\right\}$ be the associated transversal frame satisfying Theorem 1. We adapt notations from Section 3 and from Equation (22). Let $\mathcal{Y}$ be a Blaschke normal on $N$ and $\lambda_{1}$ be a function on $M$ such that $\xi_{2}=\lambda_{1} \xi_{1}+\mathcal{Y}$. We will also use the following notations:

$$
\begin{aligned}
& b:=h^{1}\left(X_{1}, X_{2}\right), \quad c:=h^{1}\left(X_{2}, X_{2}\right), \\
& \tau_{j k}^{i}:=\tau_{j}^{i}\left(X_{k}\right),
\end{aligned}
$$


We note that conditions Equations (24) up to (29) are satisfied with $f=1$. Therefore, we immediately obtain the following equations:

$$
\begin{aligned}
& 2 a_{1}+2 \lambda_{1} \tau_{11}^{2}=0 \\
& a_{2}+a_{3}+\lambda_{1} \tau_{12}^{2}+b \tau_{11}^{2}=0 \\
& 2 a_{4}+2 b \tau_{12}^{2}=0 \\
& 2 a_{5}+2 b \tau_{11}^{2}=0 \\
& a_{6}+a_{7}+b \tau_{12}^{2}+\left(c+\lambda_{1}\right) \tau_{11}^{2}=0 \\
& 2 a_{8}+2\left(c+\lambda_{1}\right) \tau_{12}^{2}=0 .
\end{aligned}
$$

We can derive more relations from the assumptions about the transversal bundle.

Lemma 3. The transversal bundle $\sigma$ is equiaffine if and only if

$$
\begin{aligned}
& a_{1}+a_{4}=0, \\
& a_{5}+a_{8}=0 .
\end{aligned}
$$

The transversal bundle $\sigma$ is antisymmetric if and only if

$$
\begin{aligned}
& a_{2}+a_{3}+2 a_{5}=0 \\
& a_{6}+a_{7}+2 a_{4}=0 .
\end{aligned}
$$

Proof. From Equation (18), we obtain:

$$
\begin{aligned}
& -2 g\left(\nabla_{X_{1}} X_{1}, X_{1}\right)-2 g\left(\nabla_{X_{1}} X_{2}, X_{2}\right)=0, \\
& -2 g\left(\nabla_{X_{2}} X_{1}, X_{1}\right)-2 g\left(\nabla_{X_{2}} X_{2}, X_{2}\right)=0,
\end{aligned}
$$

which is equivalent to

$$
\begin{aligned}
& 0=g\left(a_{1} X_{1}+a_{2} X_{2}, X_{1}\right)+g\left(a_{3} X_{1}+a_{4} X_{2}, X_{2}\right)=a_{1}+a_{4}, \\
& 0=g\left(a_{5} X_{1}+a_{6} X_{2}, X_{1}\right)+g\left(a_{7} X_{1}+a_{8} X_{2}, X_{2}\right)=a_{5}+a_{8} .
\end{aligned}
$$

Conditions from Equation (20), equivalent to antisymmetry of $\sigma$, yield

$$
\begin{aligned}
& -2 g\left(\nabla_{X_{2}} X_{1}, X_{1}\right)-g\left(\nabla_{X_{1}} X_{2}, X_{1}\right)-g\left(X_{2}, \nabla_{X_{1}} X_{1}\right)=0, \\
& -2 g\left(\nabla_{X_{1}} X_{2}, X_{2}\right)-g\left(\nabla_{X_{2}} X_{1}, X_{2}\right)-g\left(X_{1}, \nabla_{X_{2}} X_{2}\right)=0,
\end{aligned}
$$

which is equivalent to

$$
\begin{aligned}
& 0=2 g\left(a_{5} X_{1}+a_{6} X_{2}, X_{1}\right)+g\left(a_{3} X_{1}+a_{4} X_{2}, X_{1}\right)+g\left(X_{2}, a_{1} X_{1}+a_{2} X_{2}\right)=2 a_{5}+a_{3}+a_{2}, \\
& 0=2 g\left(a_{3} X_{1}+a_{4} X_{2}, X_{2}\right)+g\left(a_{5} X_{1}+a_{6} X_{2}, X_{2}\right)+g\left(X_{1}, a_{7} X_{1}+a_{8} X_{2}\right)=2 a_{4}+a_{6}+a_{7} .
\end{aligned}
$$

Lemma 4. If $x \in M$ is not a point of inflection then

$$
\tau_{11}^{2}=0 \text { and } \tau_{12}^{2}=0
$$

in a neighborhood of $x$.

Proof. At the beginning, we do computations at point $x$. We recall that an inflection point $b=c=0$ (see [2]). Combining Equations (38), (40) and (44), we get $\lambda_{1} \tau_{11}^{2}+b \tau_{12}^{2}=0$, and from Equations (40), (42) and (47), we get $3 b \tau_{12}^{2}+\left(c+\lambda_{1}\right) \tau_{11}^{2}=0$. After subtracting these equations, we obtain

$$
2 b \tau_{11}^{2}+c \tau_{12}^{2}=0
$$


Analogously, from Equations (41), (43) and (45), we get $b \tau_{11}^{2}+\left(c+\lambda_{1}\right) \tau_{12}^{2}=0$, and from Equations (39), (41) and (46), we get $\lambda_{1} \tau_{12}^{2}+3 b \tau_{11}^{2}=0$. We subtract last two equations and obtain

$$
-c \tau_{11}^{2}+2 b \tau_{12}^{2}=0 .
$$

The determinant of the system of Equations (49) and (50), $4 b^{2}+c^{2}$, does not vanish in a neighborhood of $x$ because $x$ is not an inflection point. Thus, $\tau_{11}^{2}=0$ and $\tau_{12}^{2}=0$ in a neighborhood of $x$.

We now assume that the cubic form $K$, defined by Equation (11), vanishes on $M$ so $K^{1}=K^{2}=0$ on $M$. After straightforward computations, applying all the triples $\left(X_{i}, X_{j}, X_{k}\right)$ to $K^{1}, K^{2}$ and also using Equations (12), (44), (45) and (48), we obtain the following lemma.

Lemma 5. If the cubic form $K$ is equal to 0 identically, then the following equations hold:

$$
\begin{aligned}
& -2 a_{2} b+\tau_{21}^{1}=0, \\
& X_{1}(b)-a_{2} c-a_{3}+\tau_{11}^{1} b=0, \\
& X_{1}(c)-2 a_{3} b-2 a_{4} c+\tau_{11}^{1} c+\tau_{21}^{1}=0, \\
& -2 a_{6} b+\tau_{22}^{1}=0, \\
& X_{2}(b)-a_{6} c-a_{7}+\tau_{12}^{1} b=0, \\
& X_{2}(c)-2 a_{7} b-2 a_{8} c+\tau_{12}^{1} c+\tau_{22}^{1}=0, \\
& -2 a_{1}+\tau_{21}^{2}=0, \\
& -a_{2}-a_{3}=0, \\
& -2 a_{4}+\tau_{21}^{2}=0, \\
& -2 a_{5}+\tau_{22}^{2}=0, \\
& -a_{6}-a_{7}=0, \\
& -2 a_{8}+\tau_{22}^{2}=0 .
\end{aligned}
$$

If we make use of Equation (44) up to Equations (47), (58)-(61), we obtain the following

\section{Corollary 1.}

$$
\begin{aligned}
& a_{1}=a_{4}=a_{5}=a_{8}=0, \\
& a_{3}=-a_{2}, a_{7}=-a_{6}, \\
& \tau_{21}^{2}=\tau_{22}^{2}=0 .
\end{aligned}
$$

We finally apply our last assumption $\nabla^{\perp} g^{\perp}=0$.

Lemma 6. If $\nabla^{\perp} g^{\perp}=0$ then

$$
\begin{aligned}
& \tau_{11}^{1}=0, \\
& \tau_{12}^{1}=0, \\
& X_{1}(c)=-2 \tau_{21}^{1}, \\
& X_{2}(c)=-2 \tau_{22}^{1}, \\
& X_{1}\left(4 b^{2}+\frac{5}{4} c^{2}\right)+c \tau_{21}^{1}=0, \\
& X_{2}\left(4 b^{2}+\frac{5}{4} c^{2}\right)+c \tau_{22}^{1}=0, \\
& X_{1}(b)=a_{2}(c-1), \\
& X_{2}(b)=a_{6}(c-1) .
\end{aligned}
$$


Proof. We have

$$
0=\left(\nabla^{\perp} g^{\perp}\right)\left(X_{1}, \xi_{1}, \xi_{1}\right)=-2 g^{\perp}\left(\tau_{11}^{1} \xi_{1}+\tau_{11}^{2} \xi_{2}, \xi_{1}\right)=-2 \tau_{11}^{1}
$$

which gives Equation (63). Similarly,

$$
0=\left(\nabla^{\perp} g^{\perp}\right)\left(X_{2}, \xi_{1}, \xi_{1}\right)=-2 g^{\perp}\left(\tau_{12}^{1} \xi_{1}+\tau_{12}^{2} \xi_{2}, \xi_{1}\right)=2 \tau_{12}^{1}
$$

which yields Equation (64). Next, we have

$$
\begin{aligned}
0 & =\left(\nabla^{\perp} g^{\perp}\right)\left(X_{1}, \xi_{1}, \xi_{2}\right)=-\frac{1}{2} X_{1}(c)-g^{\perp}\left(\tau_{11}^{1} \xi_{1}+\tau_{11}^{2} \xi_{2}, \xi_{2}\right)-g^{\perp}\left(\xi_{1}, \tau_{21}^{1} \xi_{1}+\tau_{21}^{2} \xi_{2}\right) \\
& =-\frac{1}{2} X_{1}(c)+\frac{1}{2} c \tau_{11}^{1}-\tau_{21}^{1}
\end{aligned}
$$

which, together with Equation (63) implies Equation (65). We also have

$$
\begin{aligned}
0 & =\left(\nabla^{\perp} g^{\perp}\right)\left(X_{2}, \xi_{1}, \xi_{2}\right)=-\frac{1}{2} X_{2}(c)-g^{\perp}\left(\tau_{12}^{1} \xi_{1}+\tau_{12}^{2} \xi_{2}, \xi_{2}\right)-g^{\perp}\left(\xi_{1}, \tau_{22}^{1} \xi_{1}+\tau_{22}^{2} \xi_{2}\right) \\
& =-\frac{1}{2} X_{2}(c)+\frac{1}{2} c \tau_{12}^{1}-\tau_{22}^{1}
\end{aligned}
$$

which, together with Equation (64) implies Equation (66). Additionally,

$$
0=\left(\nabla^{\perp} g^{\perp}\right)\left(X_{1}, \xi_{2}, \xi_{2}\right)=X_{1}\left(4 b^{2}+\frac{5}{4} c^{2}\right)-2 g^{\perp}\left(\tau_{21}^{1} \xi_{1}+\tau_{21}^{2} \xi_{2}, \xi_{2}\right)
$$

yields Equation (67) and

$$
0=\left(\nabla^{\perp} g^{\perp}\right)\left(X_{2}, \xi_{2}, \xi_{2}\right)=X_{2}\left(4 b^{2}+\frac{5}{4} c^{2}\right)-2 g^{\perp}\left(\tau_{22}^{1} \xi_{1}+\tau_{22}^{2} \xi_{2}, \xi_{2}\right)
$$

gives Equation (68). The last two equations are a consequence of Equations (52), (55), (58), (61), (63) and (64).

Corollary 2. The coefficients $\tau_{j k}^{i}$ of the normal connection vanish for all $i, j, k=1,2$. Moreover, the function $c$ is constant.

Proof. We are left with a proof that $\tau_{21}^{1}=\tau_{22}^{1}=0$. By Equations (51) and (65), we obtain

$$
X_{1}(c)=-4 a_{2} b
$$

By Equations (54) and (66), similarly, we get

$$
X_{2}(c)=-4 a_{6} b
$$

Substituting Equations (69) and (71) into Equation (67), we get $8 b\left(a_{2}(c-1)\right)+\frac{5}{2}\left(-4 a_{2} b\right)$ $=-2 c a_{2} b$ whence

$$
a_{2} b=0
$$

and $\tau_{21}^{1}=0$ by Equation (51). Analogously,

$$
a_{6} b=0
$$

hence $\tau_{22}^{1}=0$. The last assertion is now obvious.

Lemma 7. After a suitable change of the orthonormal tangent frame function $b$ is equal to zero on the whole surface and function $c$ is a non-zero constant. Moreover, $a_{2}=a_{6}=0$ identically. 
Proof. Assume that $b\left(x_{0}\right) \neq 0$ at a point $x_{0} \in M$. Then $b \neq 0$ in a neighborhood of $x_{0}$, so Equations (73) and (74) imply that $a_{2}=a_{6}=0$ there. From Equations (69) and (70), we deduce that $b$ is constant in this neighborhood of $x_{0}$. It follows that $b$ is equal to $b\left(x_{0}\right) \neq 0$ in an open and closed set in $M$ so is in the whole of $M$. Then we apply Lemma 3.8 from [2], and we change the tangent frame in the following way (the tildas indicate entries associated with the new bases):

$$
\begin{aligned}
& \tilde{X}_{1}=\cos \theta X_{1}+\sin \theta X_{2} \\
& \tilde{X}_{2}=-\sin \theta X_{1}+\cos \theta X_{2}
\end{aligned}
$$

which, according to [2], implies that

$$
\begin{aligned}
& \tilde{b}:=\tilde{h}^{1}\left(\tilde{X}_{1}, \tilde{X}_{2}\right)=b \cos 2 \theta+\frac{1}{2} c \sin 2 \theta \\
& \tilde{c}:=\tilde{h}^{!}\left(\tilde{X}_{2}, \tilde{X}_{2}\right)=c \cos 2 \theta-2 b \sin 2 \theta .
\end{aligned}
$$

If we take $\theta \in \mathbb{R}$ such that $\cot 2 \theta=-\frac{c}{2 b}$ then Equations (77) and (78) imply $\tilde{b}=0$ and $\tilde{c}$ being a non-zero constant because there are no inflection points. Now, if $\tilde{c}=1$, we could replace $\theta$ with $\theta+\frac{\pi}{2}$ obtaining $\tilde{b}=0$ and $\tilde{c}=-1$. Thus, we can always assume that $\tilde{c}$ is never equal to 1 . Finally, by Equations (69) and (70), we have $\tilde{a}_{2}=\tilde{a}_{6}=0$ on $M$. We can also see that the lemma is true in the case when $b=0$ identically.

From now on, we will apply the assertions of Lemma 7. We introduce functions $k_{i}$ for $i=1, \ldots, 8$ such that

$$
\begin{array}{ll}
S_{1} X_{1}=k_{1} X_{1}+k_{2} X_{2}, & S_{1} X_{2}=k_{3} X_{1}+k_{4} X_{2} \\
S_{2} X_{1}=k_{5} X_{1}+k_{6} X_{2}, & S_{2} X_{2}=k_{7} X_{1}+k_{8} X_{2} .
\end{array}
$$

Lemma 8. Functions $k_{i}$ satisfy the following relations:

$$
\begin{aligned}
& k_{2}=k_{3}=k_{6}=k_{7}=k_{8}=0, k_{5}=-c k_{1}, \\
& X_{2}\left(k_{1}\right)=0, \quad X_{1}\left(k_{4}\right)=0 .
\end{aligned}
$$

Proof. We consider first the equations of Ricci. From Equation (7), we get $0=h^{1}\left(X_{1}, S_{1} X_{2}\right)-$ $h^{1}\left(X_{2}, S_{1} X_{1}\right)=-c k_{2}$, so $k_{2}=0$. From Equation (8), we get $0=h^{2}\left(X_{1}, S_{1} X_{2}\right)-h^{2}\left(X_{2}, S_{1} X_{1}\right)=$ $k_{3}-k_{2}$, so $k_{3}=0$. Analogously, by Equations (9) and (10), we have $k_{6}=k_{7}=0$.

Applying the equation of Gauss (4) to triples $\left(X_{1}, X_{2}, X_{1}\right)$ and $\left(X_{1}, X_{2}, X_{2}\right)$ yields $k_{8}=0$ and $k_{5}=-c k_{1}$. We omit the straightforward computations which are simplified because the left hand sides of both equations vanish. Finally we make use of the second Codazzi Equation (6) for $S_{1}$. Since its right hand side is equal to zero and $a_{i}=0$, for all $i$ as well, we are left with $X_{1}\left(k_{3}\right) X_{1}+X_{1}\left(k_{4}\right) X_{2}-X_{2}\left(k_{1}\right) X_{1}-X_{2}\left(k_{2}\right) X_{2}=0$, which immediately yields $X_{1}\left(k_{4}\right)=0$ and $X_{2}\left(k_{1}\right)=0$.

Now, we are in a position to prove the main theorem.

Theorem 4. Let $M$ be a surface contained in a locally strictly convex hyperquadric $N$ in $\mathbb{R}^{4}$. Let $\xi$ be a metric field on $M$ such that the metric $g_{\xi}$ coincides with the Blaschke metric on $N$ restricted to $M$. Let $\sigma$ be the equiaffine antisymmetric transversal bundle with respect to $g_{\xi}$. Assume that the following conditions are satisfied:

1. M has no inflection points;

2. $\nabla^{\perp} g_{\xi} \frac{\perp}{\xi}=0$, where $\nabla^{\perp}$ is the normal connection induced by $\sigma$ and $g_{\xi}^{\perp}$ is a metric in $\sigma$ associated with $\xi$;

3. the cubic form $K$ vanishes on $M$.

Then, $M$ is locally affinely equivalent to an open part of a Clifford torus, a product of an ellipse and a hyperbola or a product of an ellipse and a parabola. 
Proof. Assumptions of the theorem allow us to choose a tangent frame $\left\{X_{1}, X_{2}\right\}$ satisfying Lemma 7. Let $\left\{\xi_{1}, \xi_{2}\right\}$ be the associated transversal frame given by Theorem 1. Fix a point $x_{0} \in M$. By Corollary 1 and Lemma 7, the Lie bracket $\left[X_{1}, X_{2}\right]$ vanishes, so there is a local parametrization $x(u, v)$ of $M$ in a neighborhood $V$ of $x_{0}$ such that $x_{u}=X_{1}$ and $x_{v}=X_{2}$. Applying Lemma 8, we can see that $k_{1}$ depends only on $u$ and $k_{4}$ depends only on $v$. We can write:

$$
\begin{aligned}
& x_{u u}=D_{X_{1}} X_{1}=\xi_{2}, \\
& x_{u v}=D_{X_{1}} X_{2}=0, \\
& x_{v v}=D_{X_{2}} X_{2}=c \xi_{1}+\xi_{2}, \\
& \left(\xi_{1}\right)_{u}=D_{X_{1}} \xi_{1}=-k_{1}(u) x_{u}, \\
& \left(\xi_{1}\right)_{v}=D_{X_{2}} \xi_{1}=-k_{4}(v) x_{v}, \\
& \left(\xi_{2}\right)_{u}=D_{X_{1}} \xi_{2}=c k_{1}(u) x_{u}, \\
& \left(\xi_{2}\right)_{v}=D_{X_{2}} \xi_{2}=0 .
\end{aligned}
$$

It follows that

$$
\begin{aligned}
& x_{u u u}=c k_{1}(u) x_{u} \\
& x_{v v v}=-c k_{4}(v) x_{v} .
\end{aligned}
$$

Since $x_{u v}=0, x$ is of the form $x(u, v)=y(u)+z(v)$. Differential equations of third order-Equations (79) and (80) are linear so there are functions $y_{1}(u), y_{2}(u), z_{1}(v), z_{2}(v)$ and vectors $C_{i}, D_{i} \in \mathbb{R}^{4}$ for $i=1,2,3$ such that $y=y_{1} C_{1}+y_{2} C_{2}+C_{3}$ and $z=z_{1} D_{1}+z_{2} D_{2}+D_{3}$. The vectors $C_{1}, C_{2}, D_{1}$ and $D_{2}$ are linearly independent because $x_{u u}, x_{v v}, x_{u u u}$ and $x_{v v v}$ are linearly independent at every point. Therefore, after possibly applying an affine transformation, the parametrization can be written in the form

$$
x(u, v)=\left(y_{1}(u), y_{2}(u), z_{1}(v), z_{2}(v)\right)
$$

for $(u, v)$ in an open rectangle $U$ such that $x(U) \subset V$. The functions $y_{1}, y_{2}$ are linearly independent, and their derivatives are also linearly independent. Otherwise, the surface would be contained in a three-dimensional affine space, and therefore, all its points would be inflection ones. The same is true for $z_{i}$. The surface is contained in a hyperquadric, so its coordinates satisfy a quadratic equation, which we write in a simplified form

$$
p(u)+q(v)+\sum_{i, j=1}^{2} a_{i j} y_{i}(u) z_{j}(v)=0 .
$$

After differentiating it with respect to $u$ and then $v$, we obtain:

$$
\sum_{j}\left(\sum_{i} a_{i j} y_{i}^{\prime}(u)\right) z_{j}^{\prime}(v)=0
$$

for every $u, v$, so for $j=1,2$ and every $u$

$$
\sum_{i} a_{i j} y_{i}^{\prime}(u)=0
$$

because $z_{1}^{\prime}$ and $z_{2}^{\prime}$ are linearly independent. Finally, $a_{i j}=0$ for every $i, j$. It implies that $p(u)$ and $q(v)$ are constants. After applying an affine transformation to $x(u, v)$, we can conclude that the equation that the surface satisfies contains only a sum of first and second powers of $y_{i}$ and $z_{i}$. It is well-known (see [7]) that the only locally strictly convex hyperquadrics in $\mathbb{R}^{4}$ can be written in the following forms:

(a) $\left(x_{1}\right)^{2}+\left(x_{2}\right)^{2}+\left(x_{3}\right)^{2}+\left(x_{4}\right)^{2}=1$ (an ellipsoid), 
(b) $x_{4}=\left(x_{1}\right)^{2}+\left(x_{2}\right)^{2}+\left(x_{3}\right)^{2}$ (an elliptic paraboloid),

(c) $\left(x_{1}\right)^{2}+\left(x_{2}\right)^{2}+\left(x_{3}\right)^{2}-\left(x_{4}\right)^{2}=-1$ (hyperboloid of two sheets).

Now, we take into account that the coordinates of our surface in a certain order satisfy one of the above equations. Since every such equation contains a sum of three squares of coordinates, two of them have to be functions of the same variable. Thus, without loss of generality, we can assume that $y_{1}^{2}+y_{2}^{2}$ is a constant. Then, there are three cases:

(i) $z_{1}^{2}+z_{2}^{2}$ is constant and $x(U)$ is affine equivalent to an open part of the Cifford torus described in Example 1;

(ii) $z_{2}-z_{1}^{2}$ is constant and $x(U)$ is affine equivalent to an open part of a product of an ellipse and a parabola given in Example 3;

(iii) $z_{1}^{2}-z_{2}^{2}$ is a constant and $x(U)$ is affine equivalent to an open part of a product of an ellipse and a hyperbola described in Example 2.

The proof of the theorem is now completed.

Funding: This research received no external funding.

Institutional Review Board Statement: Not applicable.

Informed Consent Statement: Not applicable.

Data Availability Statement: Not applicable.

Acknowledgments: The author would like to thank the referees for their valuable comments, which concerned important issues of the paper and improved the original manuscript.

Conflicts of Interest: The author declares no conflict of interest.

\section{References}

1. Ghomi, M. Strictly Convex Submanifolds and Hypersurfaces of Positive Curvature. J. Differ. Geom. 2001, 57, 239-271. [CrossRef]

2. Nuño-Ballesteros, J.J.; Sánchez, L. Affine metrics of locally strictly convex surfaces in affine 4-space. Geom. Dedicata 2016, 183, 1-24. [CrossRef]

3. Nomizu, K.; Sasaki, T. Affine Differential Geometry. Geometry of Affine Immersions; Cambridge Tracts in Mathematics; Cambridge University Press; Cambridge, UK, 1994; Volume 111.

4. Nomizu, K.; Vrancken, L. A new equiaffine theory for surfaces in $\mathbb{R}^{4}$. Int. J. Math. 1993, 4, 127-165. [CrossRef]

5. Opozda, B. A characterization of the Clifford torus. Bull. Belg. Math.-Soc.-Simon Stevin 2011, 18, 509-516. [CrossRef]

6. Opozda, B. Weak geometric structures on submanifolds of affine spaces. Tohoku Math. J. 2008, 60, 383-401. [CrossRef]

7. Li, A.M.; Simon, U.; Zhao, G.S. Global Affine Differential Geometry of Hypersurfaces; de Gruyter Expositions in Mathematics; Walter de Gruyter \& Co.: Berlin, Germany, 1993; Volume 11. 